\title{
Time-Frequency Properties of the Short-Time Linear Canonical Transform and Its Application
}

\author{
Lei Huang, ${ }^{1}$ Qingying Sun, ${ }^{1}$ Qian Xi ${ }^{1},{ }^{1}$ Yuhu Liu, ${ }^{2,3}$ Mengsheng An, ${ }^{1}$ and Zihan Zhou \\ ${ }^{1}$ School of Computer Science and Technology, Huaiyin Normal University, Huai'an 223300, China \\ ${ }^{2}$ School of Automation, Chongqing University, Chongqing 400044, China \\ ${ }^{3}$ Key Laboratory of Complex System Safety and Control, Ministry of Education, Chongqing University, Chongqing 400044, China
}

Correspondence should be addressed to Qian Xi; xiqian@hytc.edu.cn

Received 31 August 2021; Accepted 22 December 2021; Published 10 January 2022

Academic Editor: Paolo Crippa

Copyright ( $\odot 2022$ Lei Huang et al. This is an open access article distributed under the Creative Commons Attribution License, which permits unrestricted use, distribution, and reproduction in any medium, provided the original work is properly cited.

The short-time linear canonical transform (STLCT) is a novel time-frequency analysis tool, which has attracted some attention recently. However, its applications in signal processing are limited because the time-frequency properties of the STLCT are still little known. Most existing studies focus on mathematical properties rather than time-frequency properties in signal processing. To handle this problem, first, we investigate some basic time-frequency properties such as 2-D resolution of the time-frequency plane, the STLCT domain support, computation of the STLCT, etc by generalizing the short-time Fourier transform to the STLCT. Then, based on these derived properties, we find that the Gaussian window is optimal window of the STLCT. Signal separation verified the results.

\section{Introduction}

The short-time Fourier transform (STFT) has been the widely used as time-frequency analysis tool for many years. It can extract the characteristic behavior of a signal in joint time-frequency domains. Recently, the linear canonical transform (LCT), a generalization of the traditional Fourier transform (FT) and well-known fractional Fourier transform (FRFT), has received much attention in signal processing [1-8]. The LCT is more flexible because it has a total of four free parameters and one constraint. Many theoretical issues of the FT and FRFT have been extended to the LCT, which has found many research results in signal processing [9-22]. But the LCT fails in locating frequency-domain contents due to its global kernel function. For some practical applications, we want to know not only the LCT frequency contents but also how they change by time. For this reason, Kou and $\mathrm{Xu}$ [23] first systematically introduced the shorttime linear canonical transform (STLCT), in which two mathematical theorems were developed.

The motivation of this paper is to study the time-frequency properties of the STLCT, especially the optimal window selection. Since the STLCT is a generalized form of classical transformation, such as the STFT, its possible applications theoretically include nonstationary signal filtering, radar signal analysis, and so on. The premise of these applications is the property of the STLCT, such as sampling theorem, calculation algorithm, and so on [18]. To our knowledge, few achievements on time-frequency properties associated with the STLCT have been reported. Thus, we account for time-frequency properties of the STLCT from the point of view of signal processing. Serving as a novel time-frequency analysis tool, window selection and applications of the STLCT are still little known because of the following two reasons.

The first reason is most related work focused more on its mathematical theories than time-frequency properties. For instance, in [23], Kou and $\mathrm{Xu}$ proposed the STLCT and developed many useful theorems including covariance property, orthogonality property, and limit theorem in function space. Then, in [24], they also proved the Paley-Wiener theorems and the uncertainty principles for the (inverse) windowed linear canonical transform. Subsequently, the STLCT has also received attention by scholars. In [25], Zhang addressed a sampling theorem for the STLCT by means of generalized Zak transforms associated with the LCT and investigated biorthogonal condition of series 
expansion of the STLCT. Besides, Guanlei et al. discussed the uncertainty inequation relation in two STLCT domains [26]. Recently, in [27], we studied the uncertainty principle in one STLCT domain based on the definition of time width and frequency bandwidth of its time-frequency plane. Most studies paid more attention to its mathematic properties. We study time-frequency performance of the STLCT in this paper. In fact, the LCT has been introduced to classic timefrequency analysis tool, such as Wigner distribution (WD) and STFT [28-30]. For instance, in [29], Zhang and Luo considered the time-frequency tool and its application using the Gaussian windowed STFT and LCT. The performance of frequency location depends on sliding window of the STFT.

The second issue is that there are few studies on the timefrequency properties of the STLCT. As a novel time-frequency analysis tool, these basic properties are prerequisites of applications. For example, the principle of selecting the optimal window function was studied in [31]. So, we want to know the upper bound of 2-D resolution of the time-frequency plane and the minimum STLCT domain support. That is to say, how to select an appropriate window will have an impact on performance of the STLCT. For practical applications, digital computation of the STLCT is also needed to study. Besides, some other time-frequency features such as inverse STLCT, time delay, and frequency shift properties are necessary supplementary principles to its applications.

The contribution of this paper is twofold. First, we develop the time-frequency properties of the STLCT by generalizing the short-time Fourier transform (STFT) to the STLCT by substituting the FT kernel function with the LCT kernel function, including linearity, time delay and frequency shift, inverse STLCT, etc. Second, since performance of the STLCT depends largely on the window function, we prove that the Gaussian window function is optimal. The Gaussian-windowed STLCT has both maximum 2D resolution and minimum time-frequency domain support. Furthermore, performance of the STLCT affected by different windows is studied by signal separation.

Our paper is organized as follows. In Section 2, the LCT and the STLCT as well as the previous works about timefrequency analysis are reviewed. In Section 3, framework of the STLCT is established, including the idea of the STLCT, inverse STLCT, 2-D resolution of the time-frequency plane, digital computation algorithm, and STLCT domain support. In Section 4, an optimal STLCT is developed and some potential applications are discussed. In Section 5, we summarize the paper and make a future direction.

\section{Preliminaries}

2.1. Linear Canonical Transform. The linear canonical transform (LCT) of a signal $f(t)$ with parameter $\mathrm{A}$ is defined as [3]

$$
L_{A}(f)(u)= \begin{cases}\int_{-\infty}^{\infty} O_{A}(t, u) f(t) \mathrm{d} t, & b \neq 0, \\ \sqrt{d} e^{j(c d / 2) u^{2}} f(\mathrm{~d} u), & b=0,\end{cases}
$$

where $a, b, c$, and $d$ are real numbers satisfying $a d-b c=1$. The kernel function of the LCT is defined as

$$
O_{A}(t, u)=\frac{1}{\sqrt{j 2 \pi b}} e^{j / 2 b\left(a t^{2}-2 t u+d u^{2}\right)} b \neq 0
$$

The LCT satisfies the additivity property as follows:

$$
L_{(e, f, g, h)}[f(t)]=L_{\left(a_{2}, b_{2}, c_{2}, d_{2}\right)}\left[L_{\left(a_{1}, b_{1}, c_{1}, d_{1}\right)} f(t)\right],
$$

where

$$
\left[\begin{array}{ll}
e & f \\
g & h
\end{array}\right]=\left[\begin{array}{ll}
a_{2} & b_{2} \\
c_{2} & d_{2}
\end{array}\right]\left[\begin{array}{ll}
a_{1} & b_{1} \\
c_{1} & d_{1}
\end{array}\right]
$$

Thus, inverse LCT can be deduced through the additivity property, particularly,

$$
f(t)=L_{A^{-1}}\left[L_{A}(f)(u)\right]
$$

where $A^{-1}=\left[\begin{array}{c}d,-b \\ -c, a\end{array}\right]$ is the inverse of matrix. Other properties of the LCT, such as sampling, eigenfunction, uncertainty principles, fast computation, and relationships with other transforms, can be found in detail in [28].

The LCT is a generalized linear transform. When $\{a, b, c, d\}=\{\cos \alpha, \sin \alpha,-\sin \alpha, \cos \alpha\}$, it reduces to the FRFT. When $\{a, b, c, d\}=\{0,1,-1,0\}$, it becomes the classic FT. When the parameter $b=0$, the LCT is a scaling transform operation multiplying a linear frequency modulation signal, and we only consider $b \neq 0$ in our paper.

2.2. Short-Time Linear Canonical Transform. The LCT has the same limitations as the FT. It means that the LCT fails in locating the linear canonical domain frequency contents due to its global kernel function. So, in [23], Kit proposed the short-time linear canonical transform (STLCT) to handle this problem. Actually, a STLCT was mentioned in uncertainty principles study even earlier [17]. The definition of the STLCT is

$$
\operatorname{STLCT}_{f, A}(t, u)=\int_{-\infty}^{+\infty} f(\tau) g(\tau-t) O_{A}(\tau, u) \mathrm{d} \tau,
$$

where $g(\cdot)$ is the selected window function with a short time support and $O_{A}(\cdot)$ is the LCT kernel function. By definition, the result of the integral transform contains information about time $t$ and frequency $u$. So, we can know not only the STLCT frequency-domain contents but also how they change with time. The basis of the STLCT is defined as

$$
y(\tau \mid t, u)=g(\tau-t) O_{A^{-1}}(t, u) .
$$

\section{Theoretical Framework of the STLCT}

In this section, we will establish the time-frequency analysis theories of the STLCT. Firstly, basic time-frequency properties of the STLCT including inverse STLCT, time delay, and frequency shift properties are deduced. Then, in order to achieve a better implementation of the STLCT, an optimal window selection for STLCT is developed. According to the classic STFT, there are several kinds of window function such as rectangular window, Hamming window, Gaussian window, and so on. But only the Gaussian window can 
obtain the lower bound of the uncertainty principle of STFT and achieve minimum STFT domain support, which implies that Gaussian windowed FT is optimal STFT [31]. Secondly, as mentioned above, the STFT is a special case of the STLCT. So, we want to find out if these properties of the STFT can be generalized to the STLCT, and we seek the results via both mathematical proof and simulation verification.

\subsection{Time-Frequency Properties of the STLCT}

3.1.1. Property of Linearity. This property was mentioned in [23], and the STLCT is linear:

$$
\begin{aligned}
\operatorname{STLCT}_{k f_{1}+v f_{2}, A}(t, u)= & k \cdot \operatorname{STLCT}_{f_{1}, A}(t, u) \\
& +v \cdot \operatorname{STLCT}_{v f_{2}, A}(t, u),
\end{aligned}
$$

where $k$ and $v$ are arbitrary real constants. This property is convenient to process multicomponent signals and will be used in application part in this paper.

3.1.2. Time Delay and Frequency Shift Properties. STLCT is windowed LCT and can be viewed as a result of finitely repeated LCT. Thus, the time delay and frequency shift properties of the STLCT remain unchanged with the LCT, respectively. They are shown as follows:

$$
\begin{aligned}
& \operatorname{STLCT}_{f\left(t-t_{0}\right), A}(t, u)=e^{j\left(c u t_{0}-(1 / 2) a c t_{0}{ }^{2}\right)} \operatorname{STLCT}_{f(t), A}\left(t-t_{0}, u-a t_{0}\right), \\
& \operatorname{STLCT}_{f(t) e^{j v t}, A}(t, u)=e^{j\left(d v u-(1 / 2) b d v^{2}\right)} \operatorname{STLCT}_{f(t), A}(t, u-b v) . \\
& \\
& f(t)=\frac{1}{g(0)} \int_{-\infty}^{+\infty} \operatorname{STLCT}_{f, A}(t, u) O_{A^{-1}}(t, u) \mathrm{d} u .
\end{aligned}
$$

These properties can be used to deduce the case that both time delay and frequency shift occur to signal. In this case, the result is shown below.

$$
\operatorname{STLCT}_{f\left(t-t_{0}\right) e^{j v t}, A}=e^{j \Psi(u)} \operatorname{STLCT}_{f(t), A}\left(t-t_{0}, u-b v-a t_{0}\right),
$$

where

$$
\Psi(u)=\left(a v-b t_{0}\right) u+\frac{t_{0}^{2}-v^{2}}{2} a b+t_{0} v b^{2} .
$$

\subsubsection{Inverse STLCT}

1-D Inverse STLCT. We first assume that the reconstruction formula is

$$
\widehat{f}(t)=\int_{-\infty}^{+\infty} \operatorname{STLCT}_{f, A}(t, u) O_{A^{-1}}(t, u) \mathrm{d} u .
$$

Substituting (6) into (12), we obtain

$$
\widehat{f}(t)=C \int_{-\infty}^{+\infty} \int_{-\infty}^{+\infty} f(\tau) g(\tau-t) O_{A}(\tau, u) d \tau O_{A^{-1}}(t, u) \mathrm{d} u .
$$

Then, simplifying (13) yields

$$
\widehat{f}(t)=C g(0) f(t) .
$$

In order to obtain the perfect reconstruction, let $\widehat{f}(t)=f(t)$; therefore, the constant coefficient should satisfy the following requirement:

$$
C=\frac{1}{g(0)}
$$

So, the 1-D inverse STLCT is
2-D Inverse STLCT. We first assume that the reconstruction formula is

$\widehat{f}(\theta)=\int_{-\infty}^{+\infty} \int_{-\infty}^{+\infty} \operatorname{STLCT}_{f, A}(t, u) \widehat{g}(\theta-t) O_{A^{-1}}(\theta, u) \mathrm{d} t \mathrm{~d} u$.

Substituting (6) into (17) yields

$$
\begin{aligned}
\hat{f}(\theta)= & \int_{-\infty}^{+\infty} \int_{-\infty}^{+\infty} \int_{-\infty}^{+\infty} f(\tau) g(\tau-t) O_{A}(\tau, u) \mathrm{d} \tau \widehat{g}(\theta-t) \\
& O_{A^{-1}}(\theta, u) \mathrm{d} t \mathrm{~d} u
\end{aligned}
$$

Then, by simplifying (18), we have

$$
\widehat{f}(\theta)=f(\theta) \int_{-\infty}^{+\infty} g(\theta-t) \hat{g}(\theta-t) \mathrm{d} t .
$$

A simple form can be obtained through variable substitution:

$$
\widehat{f}(\theta)=f(\theta) \int_{-\infty}^{+\infty} g(t) \widehat{g}(t) \mathrm{d} t .
$$

Similarly, to ensure $\hat{f}(\theta)=f(\theta)$, the following equation should be satisfied:

$$
\int_{-\infty}^{+\infty} g(t) \widehat{g}(t) \mathrm{d} t=1
$$

Therefore, we usually choose an appropriate window function to meet the condition $(\|g(t)\|=1)$. The 2-D inverse STLCT is 


$$
\widehat{f}(\theta)=\int_{-\infty}^{+\infty} \int_{-\infty}^{+\infty} \operatorname{STLCT}_{f, A}(t, u) g(\theta-t) O_{A^{-1}}(\theta, u) \mathrm{d} t \mathrm{~d} u
$$

3.1.4. Uncertainty Principle. In time-frequency analysis field, Heisenberg's principle is a fundamental result in signal processing. Different time-frequency analysis tools such as FT and FRFT have different lower bounds. Let us s take the FT for example; a signal cannot be both narrowband and short duration, as the variances of FT pairs cannot be arbitrarily small. So, the lower bound is largely dependent upon the selected technology. The STFT has the same lower bound with the FT (Section 3, [26]). However, the STLCT includes the STFT, and we want to know not only whether the STLCT keeps the uncertainty property of its special case unchanged but also the reality verified by the simulation. Our previous study in [20] showed that lower bound of the STLCT remains the same with the LCT. Also, in this section, in order to get actual performance of the STLCT which could guide potential engineering practice, we choose the signal $s(t)=\sqrt{\left(P_{s} / 2\right)}$ $\cos \left(2 \pi f_{0} t+\pi K t^{2}\right)$, where $f_{0}=0, K=500$, and $P_{s}=2$, as our test signal. We study the Gaussian-windowed STLCT with different window lengths (see Figure 1).

3.1.5. 2-D Resolution of the STLCT. The STLCT can map the time-domain signal into the joint time and frequency domain. From this 2-D transformation, we can know not only the LCT domain frequency contents but also how they modify with time via its time-frequency plane. This plane is divided by many parallelograms while the plane of the GT is divided by numerous rectangles. The two sides of parallelogram represent the time width and the LCT domain bandwidth, respectively, based on uncertainty principle analysis in [9]. In a similar manner, they are defined by

$$
\left\{\begin{array}{l}
\Delta t_{h}^{2}=\frac{1}{E} \int_{-\infty}^{+\infty}\left(\tau-\tau_{h}\right)^{2}|y(\tau t, u)|^{2} \mathrm{~d} \tau, \\
\Delta \omega_{h}^{2}=\frac{1}{E} \int_{-\infty}^{+\infty}\left(\zeta-\zeta_{h}\right)^{2} Y_{A}(\zeta t, u)^{2} \mathrm{~d} \zeta,
\end{array}\right.
$$

where $E$ denotes the energy of signal, and specially, for an energy normalized signal, we have $E=\int_{-\infty}^{+\infty} \int_{-\infty}^{+\infty}$ $\operatorname{STLCT}_{f, A}(t, u) \mathrm{d} t \mathrm{~d} u=1 . Y_{A}(\zeta \mid t, u)$ is the LCT of $y(\tau t, u)$. Thus, the definition of $Y_{A}(\zeta t, u)$ is given as follows:

$$
Y_{A}(\zeta \mid t, u)=\int_{-\infty}^{+\infty} g(\tau-t) O_{A^{-1}}(\tau, \zeta) O_{A}(\tau, u) \mathrm{d} \tau
$$

We can rewrite the above equation by substituting the kernel function of the LCT. The computation process can be found in Appendix A.

$$
\begin{aligned}
Y_{A}(\zeta \mid t, u)= & \sqrt{j 2 \pi} e^{-\left(j / 2 b d^{\prime}\right)((\zeta-u) / b)^{2}} F[(\zeta-u) / b] \\
& O_{A}(t, \zeta) O_{A^{-1}}(t, u),
\end{aligned}
$$

where $F[(\zeta-u) / b]$ is the LCT of $g(\tau)$ with $A^{\prime}=(a-d$, $\left.b^{2}, c, d^{\prime}\right)$.
Similar to the classic uncertainty principle in FT domain, $\tau_{h}$ and $\zeta_{h}$ are the mean values of time domain and frequency domain, respectively. They are defined as

$$
\left\{\begin{array}{l}
\tau_{h}=\frac{1}{E} \int_{-\infty}^{+\infty} \tau y(\tau \mid t, u)^{2} \mathrm{~d} \tau \\
\zeta_{h}=\frac{1}{E} \int_{-\infty}^{+\infty} \zeta\left|Y_{A}(\zeta \mid t, u)\right|^{2} \mathrm{~d} \zeta .
\end{array}\right.
$$

The 2-D resolution of the STLCT is defined as

$$
\Psi=\frac{1}{\Delta t_{h} \Delta \omega_{h}} .
$$

From the definition of the resolution, the performance is affected by selected window function and free parameters of the STLCT. In [20], we have proved that the uncertainty principle of the STLCT is

$$
\Delta t_{h}^{2} \Delta \omega_{h}^{2} \geq \frac{b^{2}}{4}
$$

The equality holds if and only if Gaussian window function is selected. That is to say, the 2-D resolution can be obtained as

$$
\Psi \leq \frac{2}{|b|} .
$$

Clearly, it has an upper bound when we chose Gaussian window. We summarize this section as a theorem as follows.

Theorem 1. The upper bound of 2-D resolution of the STLCT is $2 /|b|$, which is achieved if and only if the Gaussian window is used.

3.1.6. STLCT Domain Support. It is known that classic timefrequency plane of the STFT is divided by many cell grids (see Figure 2), which is also called time-frequency cells. The two sides of the cell represent the STFT time width and band width. Moreover, each cell grid is bounded by rectangles. The coordinates of the center of rectangle are mean time and frequency, and two sides of the rectangle are the time width and bandwidth of the STFT. The product of the two sides (time-frequency product) is often defined as a STFT domain support.

The STLCT is generalized STFT. Therefore, similar to the STFT, the time width, bandwidth, mean time, and mean bandwidth in STLCT domain are defined as

$$
\begin{aligned}
& \left\{\begin{array}{l}
\Delta T_{S}^{2}=\frac{1}{P} \int_{-\infty}^{+\infty} \int_{-\infty}^{+\infty}\left(t-\bar{t}_{S}\right)^{2}\left|S T L C T_{f, A}(t, u)\right|^{2} \mathrm{~d} t \mathrm{~d} u \\
\Delta \Omega_{S, A}^{2}=\frac{1}{P} \int_{-\infty}^{+\infty} \int_{-\infty}^{+\infty}\left(u-\bar{u}_{S}\right)^{2}\left|S T L C T_{f, A}(t, u)\right|^{2} \mathrm{~d} t \mathrm{~d} u
\end{array}\right. \\
& \left\{\begin{array}{l}
\bar{t}_{S}=\frac{1}{P} \int_{-\infty}^{+\infty} \int_{-\infty}^{+\infty} t\left|\operatorname{STLCT}_{f, A}(t, u)\right|^{2} \mathrm{~d} t \mathrm{~d} u, \\
\bar{u}_{S}=\frac{1}{P} \int_{-\infty}^{+\infty} \int_{-\infty}^{+\infty} u\left|\operatorname{STLCT}_{f, A}(t, u)\right|^{2} \mathrm{~d} t \mathrm{~d} u .
\end{array}\right.
\end{aligned}
$$




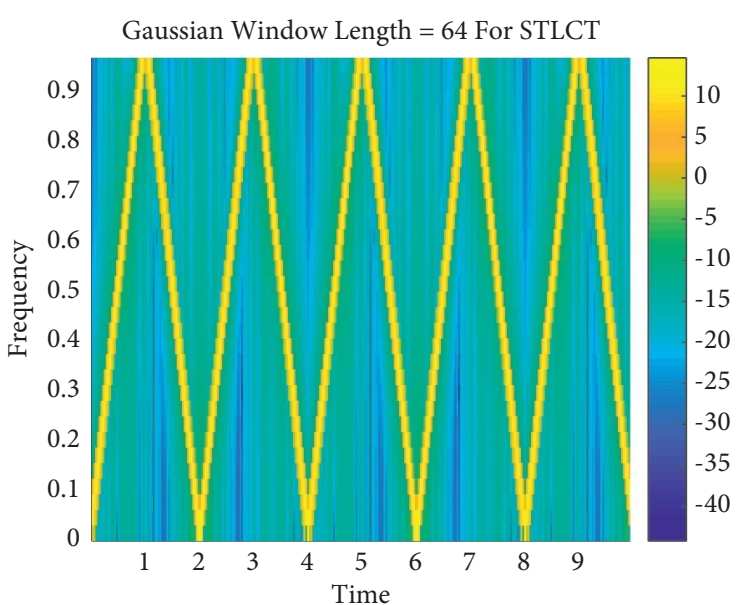

(a)

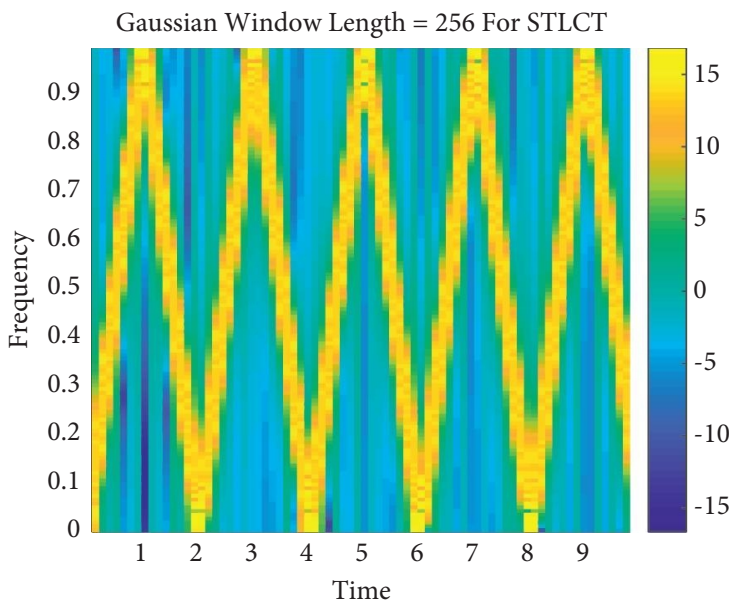

(c)

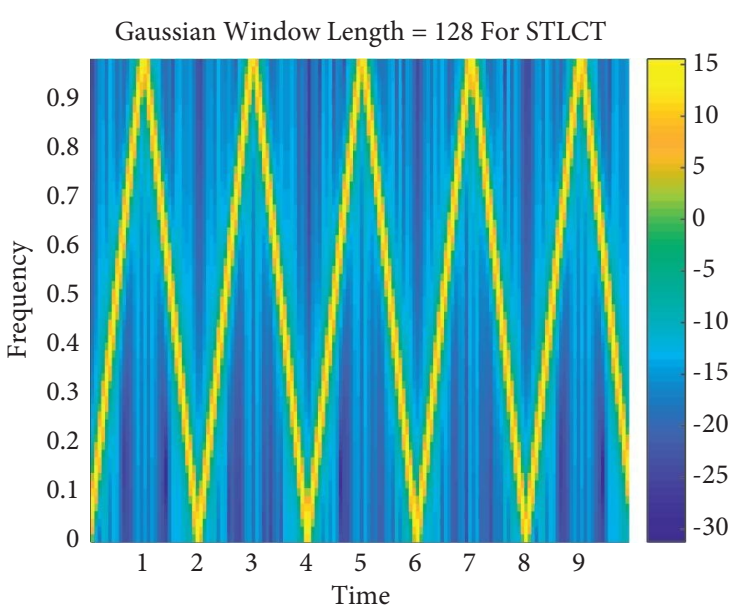

(b)

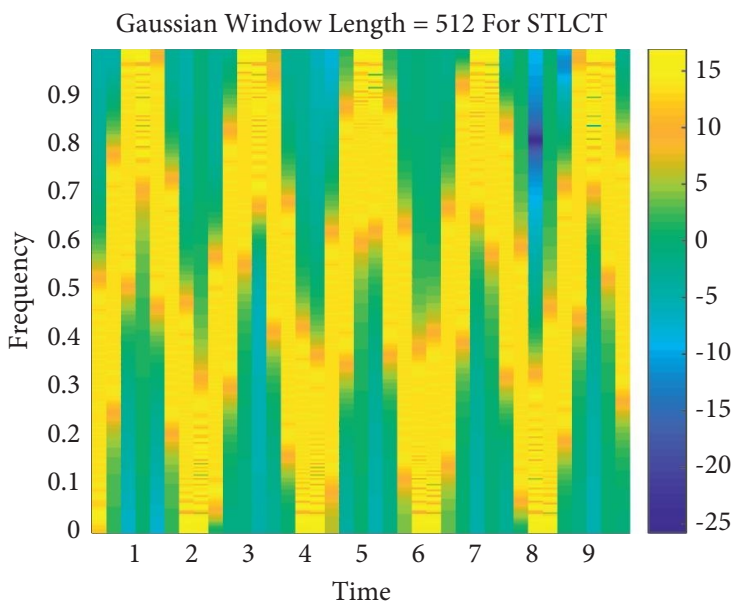

(d)

Figure 1: Performance of the Gaussian windowed STLCT with different window lengths. (a) Window length $=64$. (b) Window length $=128$. (c) Window length $=256$. (d) Window length $=512$.

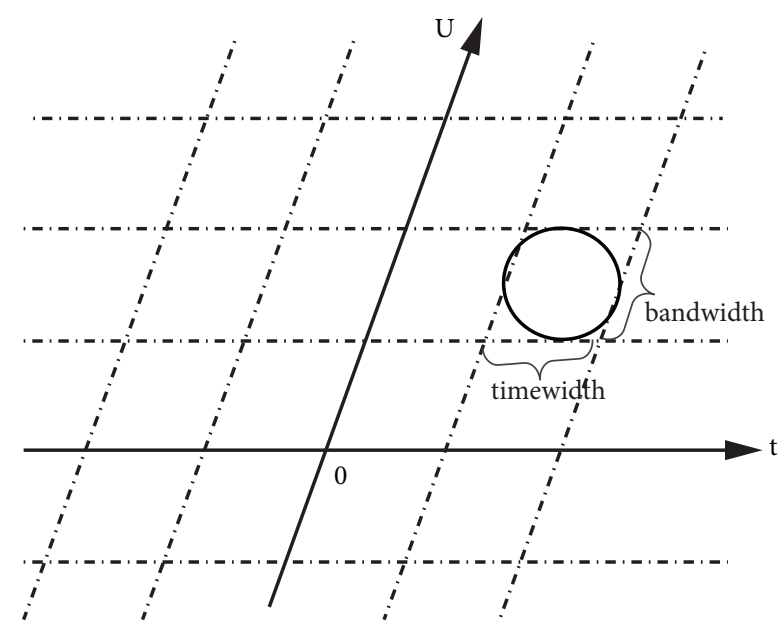

FIGURE 2: Time-frequency plane of the STLCT. The STLCT domain is bounded by many parallelograms.

To seek the relationship between the selected window function and the STLCT domain, we have the following equation, and the computation process can be found in Appendix B.

$$
\left\{\begin{array}{l}
\Delta T_{S}^{2}=\Delta T_{f}^{2}+\Delta T_{g}^{2}, \\
\Delta \Omega_{S, A}^{2}=\Delta \Omega_{f, A}^{2}+\Delta \Omega_{g}^{2} b^{2},
\end{array}\right.
$$

where $T_{g}^{2}$ and $\Omega_{g}^{2}$ are the squared time and bandwidth of the given window function. Also, the STLCT domain support is defined as squared product of $\Delta T_{S}^{2}$ and $\Delta \Omega_{S, A}^{2}$.

$$
\operatorname{TFBP}_{S, A}^{2}=\Delta T_{S}^{2} \Delta \Omega_{S, A}^{2} \text {. }
$$

3.2. Computation of the STLCT. The basic idea of computation of the STLCT is as follows. First, original signal is intercepted and captured by a sliding window in time domain. Second, the instantaneous STLCT spectrum in time domain is obtained by LCT of the signal portion captured by the window. Then, by moving the window along time domain, we can see how the frequency contents change by time. As the STLCT is based on LCT, to utilize the STLCT to the practical application, we choose a simple and efficient method of the LCT proposed in [29]. The discrete LCT and its inverse are as follows. Also, the computation steps of the STLCT are summarized as follows. 
Step 1: select an appropriate window function and initialize a set of parameters of the LCT.

Step 2: choose the length of window.

Step 3: add the window to the given signal and compute the LCT of the signal cut by the window.

Step 4: move the window along the time till the end of the signal.

From the idea of the STLCT, we can see that computation complexity of the STLCT is the same as the LCT because the STLCT is the sum of finite LCT result of each segment signal (see Figure 3).

\section{Results}

4.1. Optimal STLCT. In this section, we demonstrate that the Gaussian window is the optimal window for the STLCT. The performance of the STLCT is determined by selected window and free parameters according to the above study. In reality, we usually preset the parameters and then select a window based on the situation. The time and frequency resolution of the STLCT is constrained by the uncertainty principle, which means that a high-frequency resolution leads to a coarse time resolution and vice versa. Thus, there is a trade-off between time and frequency resolution in choosing an appropriate window for the STLCT. Window with short length is more appropriate for signals of short duration, whereas a long length window with high-frequency resolution is more appropriate for narrowband signals.

Durak and Arikan put forward a criterion of optimal window selection in [31]. Optimal windowed STLCT has both maximum $2 \mathrm{D}$ resolution and minimum time-frequency domain support. According to the time-frequency properties (5) and (6) in Section 3, we demonstrate that the Gaussian window also has the minimum STLCT domain support, which is concluded as the following theorem.

Theorem 2. The minimum STLCT domain support exists if and only if the selected window is Gaussian, and the expression of the window function is

$$
g(\tau)=\left(\pi|b| \frac{\Delta T_{f}}{\Delta \Omega_{f, A}}\right)^{-1 / 4} e^{-\left(\tau^{2} \Delta \Omega_{f, A} / 2|b| \Delta T_{f}\right)} .
$$

Proof. According to Theorem 1, the Gaussian-windowed STLCT domain has maximum 2D resolution, so we suppose an energy normalized window function

$$
g(\tau)=\left(\pi \mu^{2}\right)^{-(1 / 4)} e^{-\left(\tau^{2} / 2 \mu^{2}\right)} .
$$

Then, squared time and bandwidth of the window can be computed by integral formula $\int_{-\infty}^{+\infty} e^{-a t^{2} \pm 2 b t+c} \mathrm{~d} t=$ $\sqrt{\pi / A} e^{\left(b^{2} / a+c\right)}$. We obtain

$$
\left\{\begin{array}{l}
\Delta T_{g}^{2}=\frac{\mu^{2}}{2}, \\
\Delta \Omega_{g}^{2}=\frac{1}{2 \mu^{2}} .
\end{array}\right.
$$

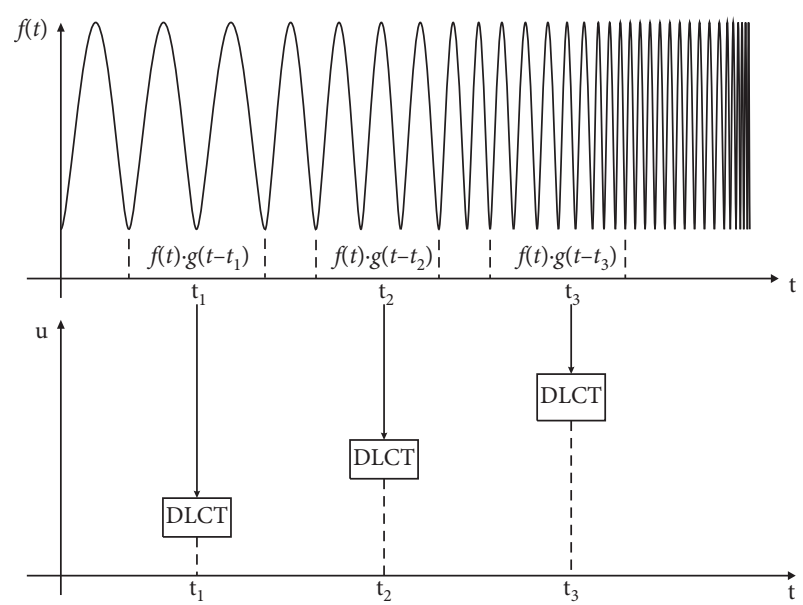

Figure 3: Schematic diagram of the short-time linear canonical transform computation. have

In addition, by substituting (30) and (33) into (31), we

$$
\begin{aligned}
\operatorname{TFBP}_{S, A}^{2} & =\left(\Delta T_{f}^{2}+\Delta T_{g}^{2}\right)\left(\Delta \Omega_{f, A}^{2}+\Delta \Omega_{g}^{2} b^{2}\right) \\
& =\Delta T_{f}^{2} \Delta \Omega_{f, A}^{2}+\Delta T_{f}^{2} \frac{b^{2}}{2 \mu^{2}}+\frac{\mu^{2}}{2} \Delta \Omega_{f, A}^{2}+\frac{\mu^{2}}{2} \frac{1}{2 \mu^{2}} b^{2} .
\end{aligned}
$$

Based on basic inequality property,

$$
\begin{aligned}
\operatorname{TFBP}_{S, A}^{2} & \geq \Delta T_{f}^{2} \Delta \Omega_{f, A}^{2}+2 \Delta T_{f} \frac{b}{\sqrt{2} \mu} \frac{\mu}{\sqrt{2}} \Delta \Omega_{f, A}+\frac{1}{4} b,^{2} \\
& =\left(\Delta T_{f} \Delta \Omega_{f, A}+\frac{1}{2} b\right)^{2} .
\end{aligned}
$$

The equality is achieved if and only if $\mu^{2}=\Delta T_{f} / \Delta \Omega_{f, A}|b|$. Furthermore, the result can be reduced to the STFT and STFRFT cases when $A=(0,1,-1,0)$ and $A=(\cos \alpha, \sin \alpha,-\sin \alpha, \cos \alpha)$, respectively.

It is known that there are some commonly used windows such as rectangular window, Hamming window, and Gaussian window. We choose the signal used in Section 3 to test the performance of the STLCT with different windows under the same parameters $(a=10, b=0.5, d=10$, and window length $=128)$. It demonstrates that the Gaussian window leads to fine resolution and concentration of the time-frequency plane, relative to other windows (see Figure 4).

4.2. Signal Separation Application. Signal separation is a vital setup in many real applications such as fault diagnosis, feature selection, and classification. In this section, the STLCT is applied to signal separation. Especially, the Gaussian window is verified the optimal window of the STLCT through the application. Here we use the nonstationary mixed signals (see Figure 5).

The simulation results show that the Gaussian window function has the best time-frequency plane resolution and 

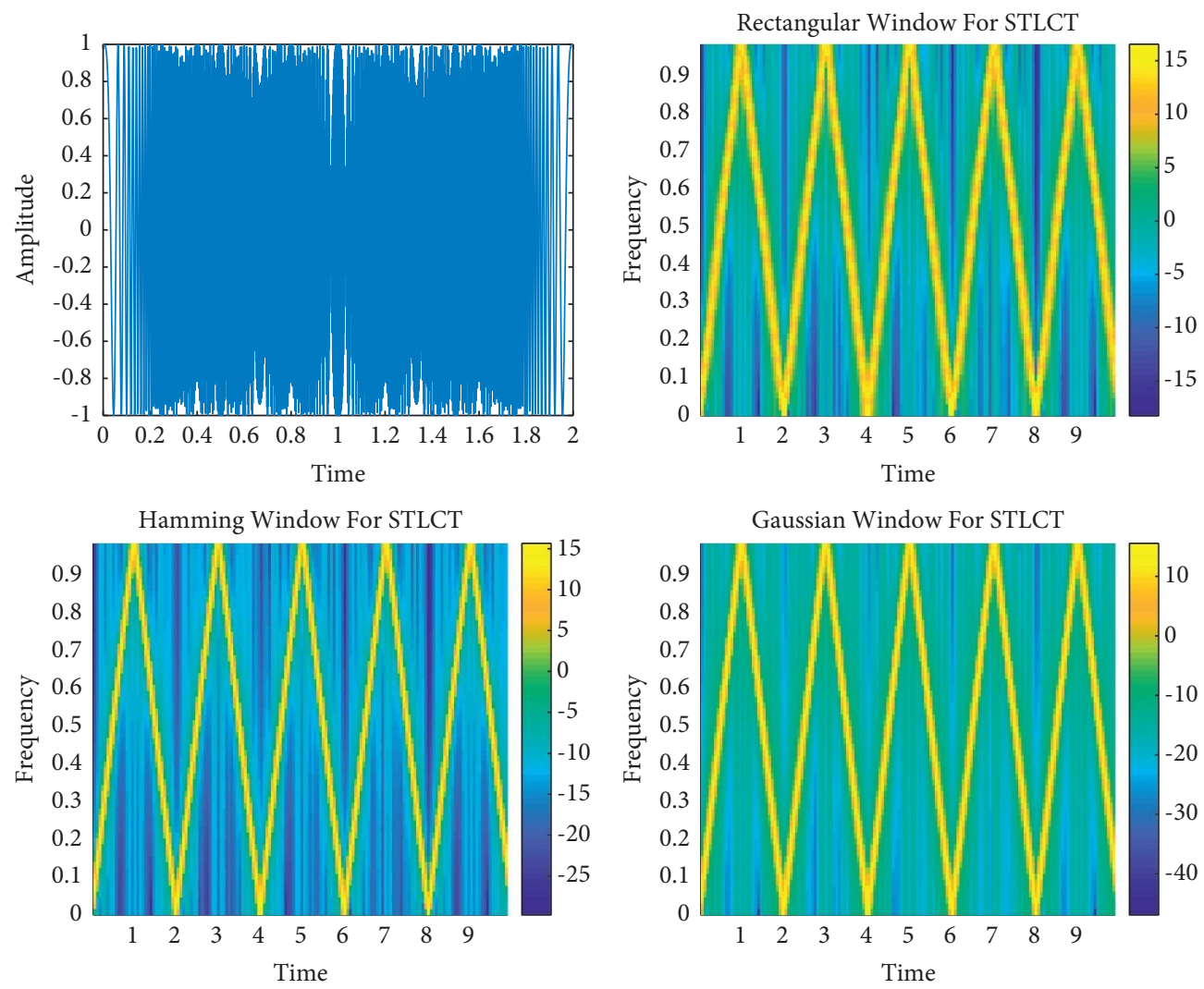

Figure 4: Performance of the STLCT with rectangular, Hamming, and Gaussian windows on the test signal.

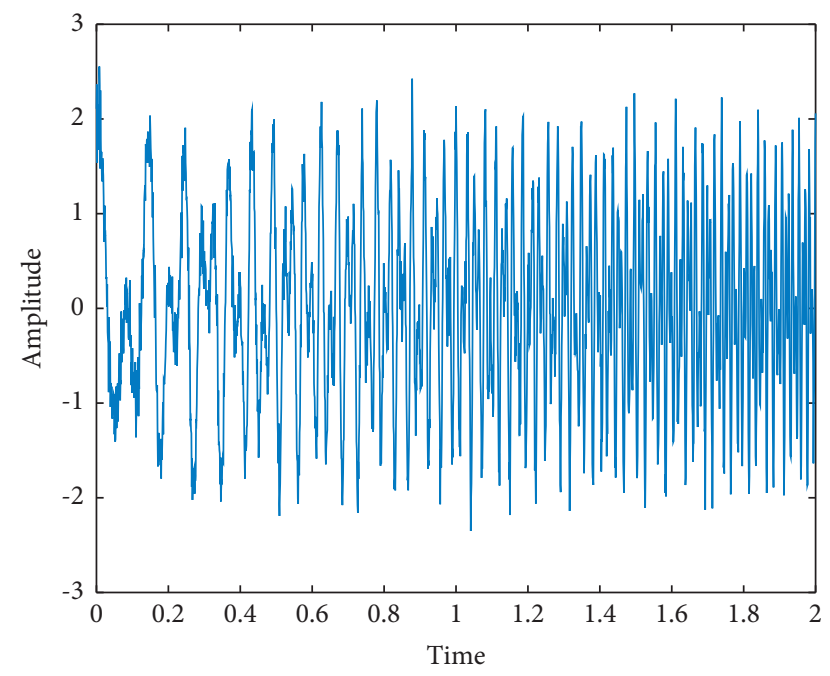

FIGURE 5: Mixed signals.

aggregation. In this section, the selective signal separation is applied to verify the superiority of Gaussian window. Signal separation is an important task in signal processing, which is often used in practical processing tasks such as filter design and fault diagnosis. For example, in fault diagnosis domain, it is often necessary to extract features from signals. For signals with fault and abnormal features, people want to separate them from normal signals for diagnosis. In this section, we will take the mixed signal of the following two nonstationary signals as an example (as shown in Figure 5) and separate them in the time-frequency plane by using the time-frequency analysis technology based on short-time linear canonical transformation.

The main steps of simulation are as follows.

Step 1: initialize the parameters of the short-term linear canonical transformation matrix and start the type and width of window function to process the original signal.

Step 2: the time-frequency distribution of the two signals is obtained by short-time linear canonical transformation.

Step 3: mask the signals to be removed to separate them.

Step 4: do the inverse transform of short-time linear canonical transform for the reserved signal.

The experimental results are shown in Figure 6. The results show that in terms of time-frequency distribution, the resolution and aggregation degree of time-frequency distribution of traditional short-time Fourier transform with window length of 256 are worse than those of short-time linear canonical transformation with window length of 512 (as shown in Figure 1). The longer the window length is, the less likely it is to reflect the frequency location characteristics theoretically. Compared with rectangular window and Hamming window, Gaussian window has the best timefrequency resolution and aggregation for short-time linear canonical transform. Especially in the third step, mask filter has better filtering effect because of its high aggregation. 

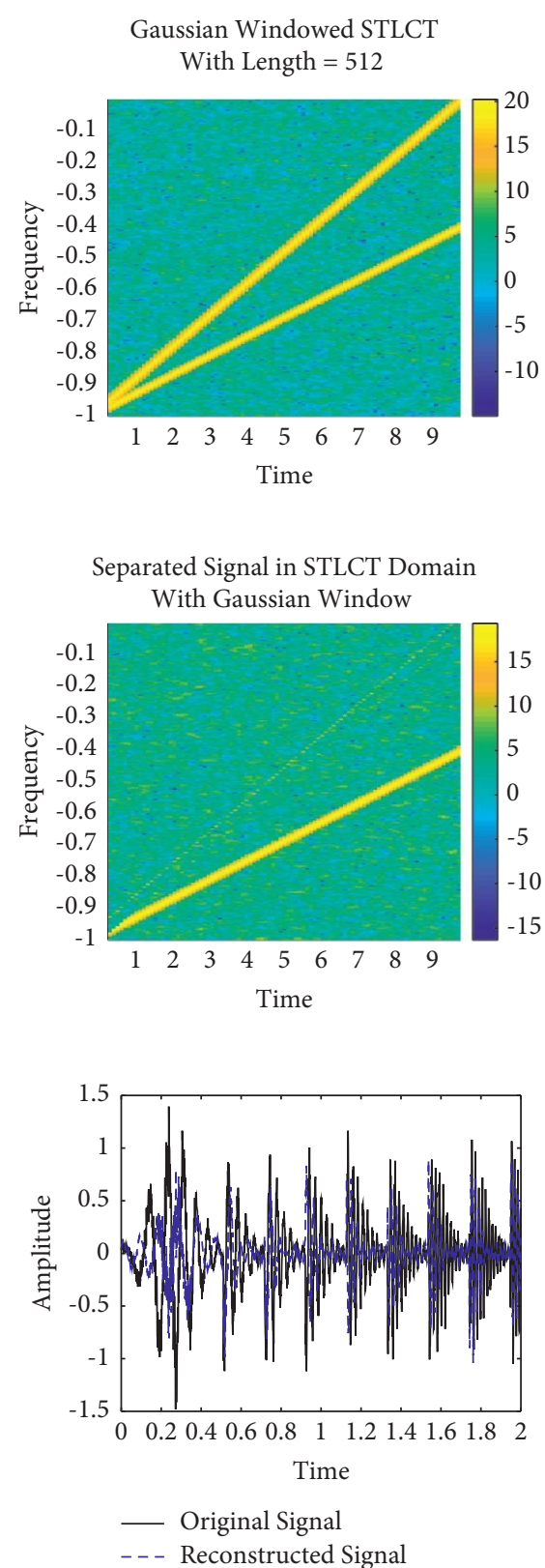

Hamming Windowed STLCT With Length $=512$

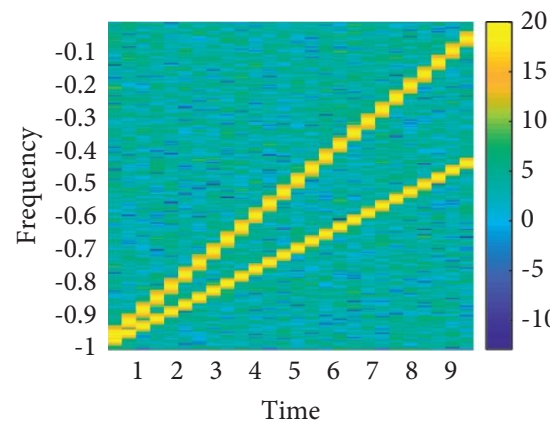

(a)

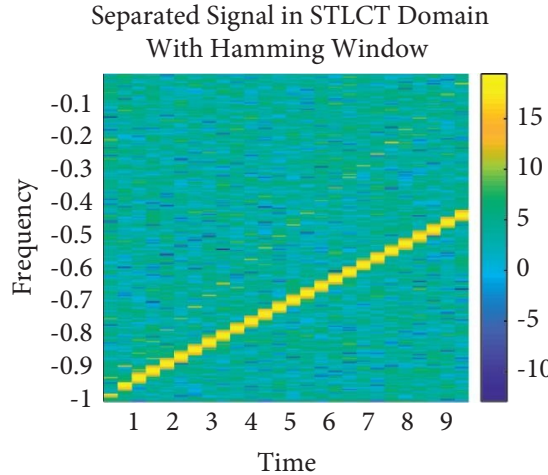

(b)

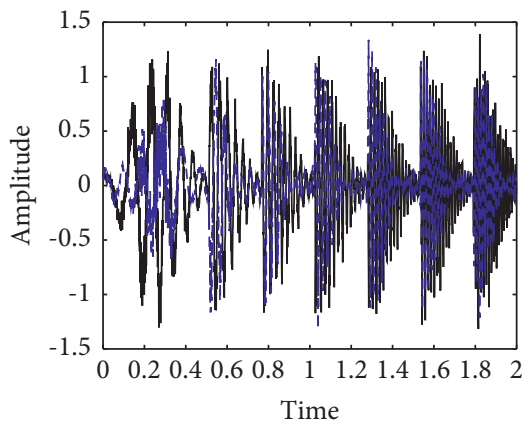

— Original Signal

- _ - Reconstructed Signal

(c)
Rectangular Windowed STLCT With Length $=512$

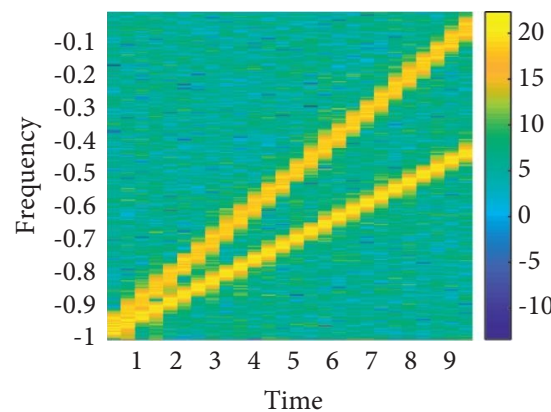

Separated Signal in STLCT Domain With Rectangular Window
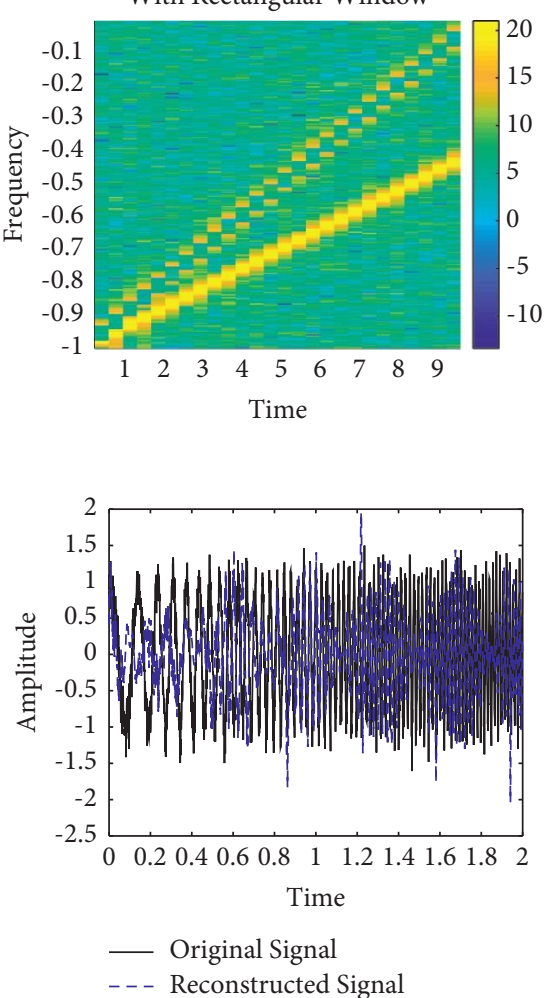

Figure 6: Comparison of signal separation performance under different windows. (a) Time-frequency distribution of the mixed signal in STLCT domain with Gaussian window, Hamming window, and rectangular window, respectively. (b) Time-frequency distribution of separated signal in STLCT domain by using mask with Gaussian window, Hamming window, and rectangular window, respectively. (c) Reconstruction of separated windowed signal with Gaussian window, Hamming window, and rectangular window, respectively.

Therefore, qualitative analysis based on simulation verifies the conclusion that Gaussian window is the optimal window function. When the parameters are given, the short-term linear canonical transform will degenerate into the traditional optimal short-time Fourier transform, namely, Gabor transform. 


\subsection{Discussion and Some Potential Applications}

4.3.1. Advantages of the STLCT. From the properties deduced in Section 3, the advantage of the STLCT is that it can obtain a higher resolution compared to the STFT and also has no cross terms in the time-frequency plane. If the Gaussian window is taken, the optimal STLCT will become the generalized Gabor transform (GT) with three free parameters. It is known that the GT has found many applications in signal analysis, image processing, and pattern recognition. As its generalized form, the STLCT has more degree of freedom.

4.3.2. Weakness of the STLCT. Although the STLCT is a new time-frequency analysis tool, it has not been used in practical scenarios so far. The classic short-time Fourier transform (STFT) (special case of the STLCT) has been widely used in the field of nonstationary signal processing. In particular, when the window function is Gaussian function, the shorttime Fourier transform is Gabor transform (GT). However, the GT did not attract scholars' interest in the decades after it was proposed. The main reason is that the expansion basis of the GT composed of window function and Fourier kernel function is nonorthogonal, which makes it difficult to calculate Gabor expansion coefficients. The STLCT is the generalized form of the STFT, so the STLCT also suffers from the difficulty of calculating its coefficients.

One of the potential applications of the STLCT is nonstationary signal filtering. Image or signal often has noise and interference. If different regions of a signal or image are contaminated with noise of different distribution characteristics, we can separate the target signal from noise in timefrequency plane based on high concentration by selecting suitable parameter matrix. We can also choose different free parameters for different regions to achieve a better performance rather than working with a fixed parameters for the whole signal or image. For example, we consider the LFM signal, the most important nonstationary signals in radar and sonar systems. Based on optimization algorithm, maximization local energy concentration can be obtained by choosing an optimal parameter matrix [14, 15]. Another potential application is in radar signal analysis. Moving target detection technology in complex background is one of the key technologies and difficulties of radar target detection, and the STFRFT has excellent performance in processing multicomponent signals, especially in high-resolution sparse domain signal processing. Since classic FRFT and STFRFT are special forms of the STLCT. Therefore, in theory, the STLCT should also have their advantages in radar target detection and parameter estimation.

4.3.3. Calculation Acceleration of the STLCT. Based on the STLCT calculation algorithm in this paper, the calculation complexity of the STLCT mainly depends on the calculation of LCT. However, the large-scale signals generated in practical engineering restrict the application of the STLCT. Fortunately, in [32, 33], Zhang et al. and Liu et al. proposed a sparse discrete FRFT algorithm to reduce the computational complexity when dealing with large signal data. Inspired by Liu et al.'s work, a feasible future direction is to study sparse LCT because LCT is the generalized form of FRFT.

\section{Conclusions}

In this paper, we have studied the time-frequency properties of the STLCT including linearity, time delay and frequency shift, inverse STLCT, computation of the STLCT, 2-D resolution, and time-frequency plane support. Gaussian window is proved to be the optimal window for the STLCT. The performance of uncertainty principle and optimal STLCT is qualitatively verified via simulation. Some potential applications also have been discussed. STLCT-based filtering design and IFE are now under investigation.

\section{Appendix}

\section{A. Computation of (26)}

$$
\begin{aligned}
Y_{A}(\zeta \mid t, u)= & \int_{-\infty}^{+\infty} g(\tau) O_{A^{-1}}(\tau+t, u) O_{A}(\tau+t, \zeta) \mathrm{d} \tau \\
= & \int_{-\infty}^{+\infty} g(\tau) \frac{1}{2 \pi b} e^{j / 2 b\left[(a-d)(\tau+t)^{2}-2 b(\tau+t)(\zeta-u)+d \zeta^{2}-a u^{2}\right]} \mathrm{d} \tau \\
= & \frac{1}{2 \pi b} e^{j / 2 b\left[a t^{2}-2 b t \zeta+d \zeta^{2}-\left(d t^{2}+2 b t u+d u^{2}\right)\right]} e^{-j / b d^{\prime}((\zeta-u) / b)^{2}} \\
& \times \int_{-\infty}^{+\infty} g(\tau) e^{-j / b(a-d) \tau t} e^{j / 2 b\left[(a-d) \tau^{2}-2 \tau b^{2} \zeta-u / b+d^{\prime}((\zeta-u) / b)^{2}\right]} \mathrm{d} \tau \\
= & \sqrt{j 2 \pi} e^{-j / 2 b d^{\prime}((\zeta-u) / b)^{2}} F\left[\frac{(\zeta-u)}{b}\right] O_{A}(t, \zeta) O_{A^{-1}}(t, u)
\end{aligned}
$$




\section{B. Proof of (31)}

To simplify (31), we first compute $P=\left\|\operatorname{STLCT}_{f, A}(t, u)\right\|^{2}$ as follows:

$$
\begin{aligned}
& \operatorname{STLCT}_{f, A}(t, u) \\
= & \int_{-\infty}^{+\infty} \int_{-\infty}^{+\infty} L_{A}(f)(\zeta) O_{A^{-1}}(\tau, \zeta) d \zeta g(\tau-t) O_{A}(\tau, u) \mathrm{d} \tau \\
= & \int_{-\infty}^{+\infty} L_{A}(f)(\zeta) \int_{-\infty}^{+\infty} g(\tau-t) O_{A^{-1}}(\tau, \zeta) O_{A}(\tau, u) \mathrm{d} \tau \mathrm{d} \zeta \\
= & \int_{-\infty}^{+\infty} L_{A}(f)(\zeta) Y_{A}(\zeta \mid t, u) \mathrm{d} \zeta .
\end{aligned}
$$

On the other hand, suppose the window function is real and symmetric, and we obtain

$$
\left|Y_{A}(\zeta \mid t, u)\right|=\frac{|F[((\zeta-u) / b)]|}{\sqrt{2 \pi} b} .
$$

So, we have the following equation:

$$
\begin{aligned}
\left\|\operatorname{STLCT}_{f, A}(t, u)\right\|^{2} & =\int_{-\infty}^{+\infty} \int_{-\infty}^{+\infty}\left|L_{A}(f)(\zeta)\right|^{2} \\
& \left|\frac{F[(u-\zeta) / b]}{\sqrt{2 \pi} b}\right|^{2} \mathrm{~d} \zeta \mathrm{d} u .
\end{aligned}
$$

By variable substitution,

$$
\left\{\begin{array}{l}
\zeta=\rho \\
u=\rho+\eta b
\end{array}\right.
$$

The Jacobian partial derivative is

$$
J=\frac{\partial(\zeta, u)}{\partial(\rho, \eta)}=\left|\begin{array}{ll}
1 & 0 \\
1 & b
\end{array}\right|=b .
$$

Then, (38) can be rewritten as

$$
\left\|\operatorname{STLCT}_{f, A}(t, u)\right\|^{2}
$$

$$
\begin{aligned}
& =\frac{1}{2 \pi b^{2}} \int_{-\infty}^{+\infty} \int_{-\infty}^{+\infty}\left|L_{A}(f)(\zeta)\right|^{2}\left|F\left[\frac{(\zeta-u)}{b}\right]\right|^{2} \mathrm{~d} \zeta \mathrm{d} u \\
& =\frac{1}{2 \pi b^{2}} \int_{-\infty}^{+\infty} \int_{-\infty}^{+\infty}\left|L_{A}(f)(\rho)\right|^{2}|F(\eta)|^{2}|J| d \rho d \eta
\end{aligned}
$$

$$
\begin{aligned}
& =\frac{1}{2 \pi b} \int_{-\infty}^{+\infty}\left|L_{A}(f)(\rho)\right|^{2} d \rho \int_{-\infty}^{+\infty}|F(\eta)|^{2} d \eta \\
& =\frac{1}{2 \pi b}\left\|L_{A}(f)(\rho)\right\|^{2}\|F(\eta)\|^{2} .
\end{aligned}
$$

Thus, $\bar{u}_{S}$ and $\Delta \Omega_{S, A}^{2}$ are obtained:

$$
\begin{aligned}
\bar{u}_{S}= & \frac{1}{\left\|\operatorname{STLCT}_{f, A}(t, u)\right\|^{2}} \int_{-\infty}^{+\infty} \int_{-\infty}^{+\infty} u\left|\operatorname{STLCT}_{f, A}(t, u)\right|^{2} \mathrm{~d} t \mathrm{~d} u \\
= & \frac{1}{\left\|\operatorname{STLCT}_{f, A}(t, u)\right\|^{2}} \int_{-\infty}^{+\infty} \int_{-\infty}^{+\infty}(\rho+b \eta)\left|L_{A}(f)(\rho)\right|^{2}\left|\frac{F(\eta)}{\sqrt{2 \pi} b}\right|^{2}|J| \mathrm{d} \rho \mathrm{d} \eta \\
= & \frac{1}{\left\|L_{A}(f)(\rho)\right\|^{2}} \int_{-\infty}^{+\infty} \rho\left|L_{A}(f)(\rho)\right|^{2} \mathrm{~d} \rho \\
& +\frac{b}{\|F(\eta)\|^{2}} \int_{-\infty}^{+\infty} \eta|F(\eta)|^{2} \mathrm{~d} \rho \mathrm{d} \eta \\
= & \bar{u}_{f}+\bar{N}_{g} b \\
\Delta \Omega_{S, A}^{2}= & \frac{1}{P} \iint\left(u-\bar{u}_{s}\right)^{2} \mid \operatorname{STLCT} \\
= & \frac{2 \pi b}{\left\|L_{A}(f)(\rho)\right\|^{2}\|F(\eta)\|^{2}} \iint\left(u-\bar{u}_{s}\right)^{2}\left|L_{A}(f)(\rho)\right|^{2}\left|\frac{F(\eta)^{2}}{\sqrt{2 \pi} b}\right||J| \mathrm{d} \rho \mathrm{d} \eta \\
= & \frac{1}{\left\|L_{A}(f)(\rho)\right\|^{2}} \int_{-\infty}^{+\infty}\left(\rho-\bar{u}_{f}\right)^{2}\left|L_{A}(f)(\rho)\right|^{2} \mathrm{~d} \rho \\
& +\frac{b^{2}}{\|F(\eta)\|^{2}} \int_{-\infty}^{+\infty}\left(\eta-\bar{N}_{g}\right)^{2}|F(\eta)|^{2} d \eta \\
= & \Delta \Omega_{f, A}^{2}+\Delta \Omega_{g}^{2} b^{2} .
\end{aligned}
$$




\section{Data Availability}

The selected signal and parameter setting data are included within the article.

\section{Conflicts of Interest}

The authors declare that they have no conflicts of interest.

\section{Acknowledgments}

This study was supported by the National Natural Science Foundation of China (62006093) and Natural Science Research Project of the Higher Education Institutions of Jiangsu Province, China (18KJB510006).

\section{References}

[1] L. Cohen, "The uncertainty principles of windowed wave functions," Optics Communications, vol. 179, no. 1-6, pp. 221-229, 2000.

[2] X.-L. Fan, K. I. Kou, and M.-S. Liu, "Quaternion Wigner-Ville distribution associated with the linear canonical transforms," Signal Processing, vol. 130, pp. 129-141, 2017.

[3] Q. Feng and B. Z. Li, "Convolution and correlation theorems for the two-dimensional linear canonical transform and its applications," IET Signal Processing, vol. 10, no. 2, pp. 125-132, 2016.

[4] M. Moshinsky and C. Quesne, "Linear canonical transformations and their unitary representations," Journal of Mathematical Physics, vol. 12, no. 8, pp. 1772-1780, 1971.

[5] J. Shi, X. Liu, L. He, M. Han, Q. Li, and N. Zhang, "Sampling and reconstruction in arbitrary measurement and approximation spaces associated with linear canonical transform," IEEE Transactions on Signal Processing, vol. 64, no. 24, pp. 6379-6391, 2016.

[6] D. Wei and Y.-M. Li, "Convolution and multichannel sampling for the offset linear canonical transform and their applications," IEEE Transactions on Signal Processing, vol. 67, no. 23, pp. 6009-6024, 2019.

[7] D. Wei and Y. Shen, "Fast numerical computation of two-dimensional non-separable linear canonical transform based on matrix decomposition," IEEE Transactions on Signal Processing, vol. 69, pp. 5259-5272, 2021.

[8] W.-B. Gao and B.-Z. Li, "The octonion linear canonical transform: definition and properties," Signal Processing, vol. 188, no. 1, Article ID 108233, 2021.

[9] Z.-C. Zhang, "Uncertainty principle for linear canonical transform using matrix decomposition of absolute spread matrix," Digital Signal Processing, vol. 89, pp. 145-154, 2019.

[10] Z.-J. Huang, S. Cheng, L.-H. Gong, and N.-R. Zhou, "Nonlinear optical multi-image encryption scheme with two-dimensional linear canonical transform," Optics and Lasers in Engineering, vol. 124, Article ID 105821, 2020.

[11] S. H. Liu, Z. Zeng, Y. D. Zhang, and T. Fan, "Automatic human fall detection in fractional Fourier domain for assisted living," in Proceedings of the 41st IEEE Int. Conf. Acoust. Speech Signal Process. (ICASSP 2016), pp. 799-803, Shanghai, China, March 2016.

[12] G. L. Xu, X. G. Xu, and X. T. Wang, “Generalized Heisenberg uncertainty relations for complex-valued signals out of Hilbert transform associated with LCT," Optik - International
Journal for Light and Electron Optics, vol. 224, Article ID 165529, 2020.

[13] Z.-C. Zhang, "Variance analysis of linear canonical Wigner distribution," Optik, vol. 212, Article ID 164633, 2020.

[14] Q. Feng, B.-Z. Li, and J.-M. Rassias, "Weighted HeisenbergPauli-Weyl uncertainty principles for the linear canonical transform," Signal Processing, vol. 165, pp. 209-221, 2019.

[15] D. Wei, W. Yang, and Y.-M. Li, "Lattices sampling and sampling rate conversion of multidimensional bandlimited signals in the linear canonical transform domain," Journal of the Franklin Institute, vol. 356, no. 13, pp. 7571-7607, 2019.

[16] X. Su, R. Tao, and X. Kang, "Analysis and comparison of discrete fractional Fourier transforms," Signal Processing, vol. 160, pp. 284-298, 2019.

[17] W. B. Gao and B. Z. Li, "Uncertainty principles for the short-time linear canonical transform of complex signals," Signal Processing, vol. 111, Article ID 102953, 2021.

[18] D. Wei and H. Hu, "Theory and applications of short-time linear canonical transform," Digital Signal Processing, vol. 118, Article ID 103239, 2021.

[19] S. Xu, L. Feng, Y. Chai, and Y. He, "Analysis of a-stationary random signals in the linear canonical transform domain," Signal Processing, vol. 146, pp. 126-132, 2018.

[20] J. Shi, X. Liu, F. Yan, and W. Song, "Error analysis of reconstruction from linear canonical transform based sampling," IEEE Transactions on Signal Processing, vol. 66, no. 7, pp. 1748-1760, 2018.

[21] J. Shi, X. Liu, Y. Zhao, S. Shi, X. Sha, and Q. Zhang, "Filter design for constrained signal reconstruction in linear canonical transform domain," IEEE Transactions on Signal Processing, vol. 66, no. 24, pp. 6534-6548, 2018.

[22] L. Zhao, J. T. Sheridan, and J. J. Healy, "Unitary algorithm for nonseparable linear canonical transforms applied to iterative phase retrieval," IEEE Signal Processing Letters, vol. 24, no. 6, pp. 814-817, 2017.

[23] K.-I. Kou and R.-H. Xu, "Windowed linear canonical transform and its applications," Signal Processing, vol. 92, no. 1, pp. 179-188, 2012.

[24] K.-I. Kou, R.-H. Xu, and Y.-H. Zhang, "Paley-wiener theorems and uncertainty principles for the windowed linear canonical transform," Mathematical Methods in the Applied Sciences, vol. 35, no. 17, pp. 2122-2132, 2012.

[25] Z.-C. Zhang, "Sampling theorem for the short-time linear canonical transform and its applications," Signal Processing, vol. 113, pp. 138-146, 2015.

[26] X. Guanlei, W. Xiaotong, and X. Xiaogang, "Uncertainty inequalities for linear canonical transform," IET Signal Processing, vol. 3, no. 5, pp. 392-402, 2009.

[27] L. Huang, K. Zhang, Y. Chai, and S. Xu, "Uncertainty principle and orthogonal condition for the short-time linear canonical transform," Signal, Image and Video Processing, vol. 10, no. 6, pp. 1177-1181, 2016.

[28] S.-C. Pei and J.-J. Ding, "Fractional Fourier transform, Wigner distribution, and filter design for stationary and nonstationary random processes," IEEE Transactions on Signal Processing, vol. 58, no. 8, pp. 4079-4092, 2010.

[29] Z. C. Zhang and M. K. Luo, "Relations between gabor transform and linear canonical transform and their application for filter design," Acta Electronica Sinica, vol. 12, p. 2525, Article ID 252943, 2015.

[30] Q. Xiang and K. Y. Qin, “A time-frequency analysis method based on linear canonical transform and short-time Fourier transform," Acta Electronica Sinica, vol. 39, no. 7, pp. 1508-1513, 2011. 
[31] L. Durak and O. Arikan, "Short-time Fourier transform: two fundamental properties and an optimal implementation," IEEE Transactions on Signal Processing, vol. 51, no. 5, pp. 1231-1242, 2003.

[32] H. Zhang, T. Shan, S. Liu, and R. Tao, "Optimized sparse fractional Fourier transform: principle and performance analysis," Signal Processing, vol. 174, Article ID 107646, 2020.

[33] S. Liu, T. Shan, R. Tao et al., "Sparse discrete fractional Fourier transform and its applications," IEEE Transactions on Signal Processing, vol. 62, no. 24, pp. 6582-6595, 2014. 